\title{
Evaluation and treatment of neurobehavioral complications of traumatic brain injury - Have we made any progress?
}

Many individuals with mild traumatic brain injury (TBI) and virtually all individuals who survive moderate and severe TBI are left with significant long-term neurobehavioral sequelae [1-3]. The reduction in TBIassociated mortality rates over the last two decades [4] has led to a significant increase in the number of individuals with long-term neurobehavioral disorders related to TBI $[5,6]$. In addition to the agitation, aggression, and confusion often seen in the acute recovery period, a variety of studies has confirmed that TBI confers a significant increase in the relative risk of developing many psychiatric disorders including mood disorders, psychotic disorders, anxiety disorders, obssesivecompulsive disorder and others $[7,8]$. TBI may well be a critical risk factor in the development of schizophrenia in those with a genetic vulnerability to this illness [9].

Even apart from formal psychiatric disorders, behavioral challenges abound. Persistent postconcussive symptoms after mild TBI have been a focus of controversy for over 100 years. Syndromes such as apathy, disorders of impulse control and affect, though perhaps not meeting formal criteria for specific psychiatric disorders, are a source of enormous excess disability. Although we must be cognizant of the critical role that environmental and psychosocial factors play in the genesis and maintenance of these neurobehavioral problems, we must also be clear that regional brain injury plays an equally critical role in the etiology of these acquired psychiatric disorders. The phenomenology of the post-TBI depressive or psychotic syndrome may be altered by the cognitive, or speech and language deficits that are present. However, the core clinical features of these disorders, their natural history, and apart from a heightened susceptibility to medication side effects, their response (or lack of response) to conventional and newer treatment interventions do not appear to differ significantly from idiopathic psychiatric disorders. Thus the assessment and treatment of the neurobehavioral sequelae TBI in many ways presents a model for studying acquired psychiatric illness, and has much to offer us in terms of understanding regional brain-behavior relationships. The confluence of knowledge gained from the study of the psychosocial effects of injury, the impact of injury acutely and over time on neurons and their supporting tissues, the effects of injury on the synapse and its implications for medication intervention, and the clarification of the typical regional brain injury profile associated with TBI suggest a new paradigm of sorts; a neurobiopsychosocial paradigm.

To the TBI survivor and those who care for him or her, changes in personality, mood, emotional stability, and impulse control are among the most devastating consequences of the injury. Assessment techniques and interventions for these sequelae have lagged behind those in other areas of neurorehabilitation. Various assistive and prosthetic devices can often improve the quality of life and the level of function in an individual with sensorimotor deficits. For the individual at the mercy of recurrent rage attacks, major mood disorders, psychotic syndromes, apathy, and predictably unpredictable displays of pathological affect there are no equivalent assistive and prosthetic devices. Behavioral, environmental, and psychopharmacological approaches can often seem a somewhat "low-tech", insufficient arsenal in the face of these challenges.

On a more positive note, the challenges presented in these disorders has led to an enormous multidisciplinary effort directed towards understanding TBI 
and its sequelae. Our knowledge of the neuropathophysiology of TBI has increased dramatically over the last decade (see [10]). Advances in neuroimaging, neuropsychology, and other neurodiagnostic techniques make possible a much finer resolution of brain injury profile associated with specific neuropsychiatric sequelae. Although there is a long way to go, what we now know about many of these behavioral challenges dwarfs what we knew just ten years ago, and sets the stage for interventions and progress for the next ten years. In this issue, we attempt to summarize what is now known about the underlying pathophysiology of several of the common neurobehavioral sequelae of TBI, explore the links between this pathophysiology and the typical profile of injury seen in TBI (in other words, "why are these behaviors so common after TBI?"), and offer a review of current thinking about practical interventions and treatments. We hope this will contribute both to the theoretical and the clinical/practical understanding of these challenges, and improve the care of individuals with TBI.

Thomas W. McAllister, M.D. Guest Editor

Dartmouth Medical School

Department of Psychiatry

Dartmouth Hitchcock Medical Center

One Medical Center Drive

Lebanon, NH 03756, USA

Tel.: +1 6036505824

Fax: +1 6036505842

E-mail: Thomas.W.McAllister@Dartmouth.edu

\section{References}

[1] DHHS, Department of Health and Human Services: Interagency Head Injury Task Force Report, US Department of Health and Human Services, Washington, DC, 1989.

[2] H.S. Levin et al., Neurobehavioral outcome 1 year after severe head injury: experience of the traumatic coma data bank, $J$ Neurosurg 73 (1990), 699-709.

[3] S.B. Sorenson and J.F. Kraus, Occurrence, severity, and outcome of brain injury, Journal of Head Trauma Rehabilitation 5 (1991), 1-10.

[4] D.M. Sosin, J. Sniezek and D. Thurman, Incidence of mild and moderate brain injury in the United States, 1991, Brain Injury 10 (1996), 47-54.

[5] D.B. Arciniegas, J. Topkoff and J.M. Silver, Neuropsychiatric aspects of traumatic brain injury, Current Treatment Options in Neurology 2(2) (2000), 169-186.

[6] T.W. McAllister, Neuropsychiatric sequelae of head injuries, Psychiatric Clinics of North America 15(2) (1992), 395-413.

[7] R. van Reekum, T. Cohen and J. Wong, Can traumatic brain injury cause psychiatric disorders? Journal of Neuropsychiatry and Clinical Neuroscience 12 (2000), 316-327.

[8] J. Silver et al., Association between severe head injuries and psychiatric disorders: findings from the New Haven NIMH epdemiologic catchment area study, Journal of Neuropsychiatry and Clinical Neuroscience 9 (1997), 640.

[9] D. Malaspina et al., Traumatic brain injury and schizophrenia in members of schizophrenia and bipolar disorder pedigrees, American Journal of Psychiatry 158 (2001), 440-446.

[10] T.A. Gennarelli and D.I. Graham, Neuropathology of the Head Injuries, Semin Clin Neuropsychiatry 3(3) (1988), 160-175. 